\title{
Pleural synovial sarcoma patient treated with combined chemotherapy and Endostar, plus sunitinib maintenance therapy: A case report and review of the literature
}

\author{
CHONG TENG $^{1 *}$, LI LI ${ }^{1 *}$, WEIXI SHEN ${ }^{1}$, JINGANG LI ${ }^{2}$, YING LIU ${ }^{1}$, QIUYING JIANG ${ }^{1}$, TAO XIN ${ }^{1}$, \\ DAYONG HUANG ${ }^{1}$, XIAOWEI SONG ${ }^{1}$, YANJU LV ${ }^{1}$ and YINGHUA JIN ${ }^{1}$ \\ ${ }^{1}$ Department of Oncology, The Second Affiliated Clinical Hospital of Harbin Medical University, Harbin, \\ Heilongjiang 150086; ${ }^{2}$ Heilongjiang Academy of Chinese Medicine, Harbin, Heilongjiang 150001, P.R. China
}

Received September 30, 2014; Accepted May 12, 2015

DOI: $10.3892 / \mathrm{ol} .2015 .3311$

\begin{abstract}
Synovial sarcoma is a rare, highly malignant soft-tissue tumor that occurs primarily in the extremities. At present, there is no effective clinical treatment for this condition. The present study reports the case of a 49-year-old male who was diagnosed with pleural synovial sarcoma and treated with recombinant human endostatin (Endostar) combined with chemotherapy for a total of six cycles, followed by sunitinib maintenance therapy. To the best of our knowledge, this is the first reported use of sunitinib for maintenance therapy in pleural synovial sarcoma. The patient survived for 25 months after the recurrence of the disease following surgery. The results indicate that this combination therapy was effective in the treatment of pleural synovial sarcoma. The present study also briefly reviews the literature on pleural synovial sarcoma, and the features and treatments for this rare case are discussed.
\end{abstract}

\section{Introduction}

Synovial sarcoma is a malignant tumor that accounts for $10 \%$ of all soft-tissue tumors (1). In total, $<3$ cases per million individuals are diagnosed each year. The disease can occur at any age, but is more common in teenagers and young adults. Despite its name, synovial sarcoma does not develop in the synovial tissues of the joints. The disease mostly presents in the extremities, particularly the periarticular regions.

Correspondence to: Professor Qiuying Jiang, Department of Oncology, The Second Affiliated Clinical Hospital of Harbin Medical University, 246 Xuefu Street, Harbin, Heilongjiang 150086, P.R. China

E-mail: jqyBM1234@163.com

*Contributed equally

Key words: pleura, synovial sarcoma, chemotherapy, Endostar, sunitinib
However, synovial sarcoma is also found in the head and neck, mediastinum, heart, esophagus, lungs, abdominal wall, mesentery, forearms, thighs, and muscle and fascia of the waist and back (1-5). In 1996, Gaertner et al reported the first case of synovial sarcoma in the literature (3). The diagnosis of synovial sarcoma is difficult due to its rarity and the fact that its clinical and histological characteristics are similar to other spindle cell pleural tumors. Histopathology and/or immunohistochemistry investigations are essential to diagnose synovial sarcoma; the diagnosis in the present study was based on immunohistochemistry, but cytogenetics can be used to detect chromosomal translocations specific to synovial sarcoma, which aids in the confirmation of the diagnosis (2). Cytogenetic studies are useful for differential diagnosis. Chromosomal translocation (chromosome $\mathrm{X}$ or 18) is observed in $>90 \%$ of synovial sarcomas and may be detected by the FISH method (fluorescence in-situ hybridization) w5x (2). In spite of its high sensitivity, the FISH method is not obligatory for the diagnosis, and it is indicated in cases of histological and inmunohistochemical overlapping.

The main poor prognostic factors are complete resection, a tumor size $>5 \mathrm{~cm}$, the male gender, a high-grade tumor and an age of $>20$ years. The patient in the present study did not undergo a complete resection due to the presence of a giant tumor. These factors are likely reasons for the disease progressing in a relatively short period of time.

\section{Case report}

A 49-year-old male was admitted on October 5 2010, to the Harbin Thoracic Hospital due to left-sided chest pain that had persisted for 1 month. Computed tomography (CT) chest scans showed bilateral pleural effusion. Between October 2010 and August 2011, the patient was treated with anti-inflammatory therapy 7 times for a duration of 2 weeks (penicillin, $4.5 \mathrm{mg}$, twice a day intravenous drops; quinolines, $100 \mathrm{ml}$, once per day intravenous drops) and underwent pleural drainage surgery, which caused a subsequent partial remission. The patient underwent an evacuation of a hematoma on April 28, 2011, as CT showed a huge low-density mass in the left pleural space (Fig. 1A-C). The histopathological specimen showed (left pleural) fibrous wall 

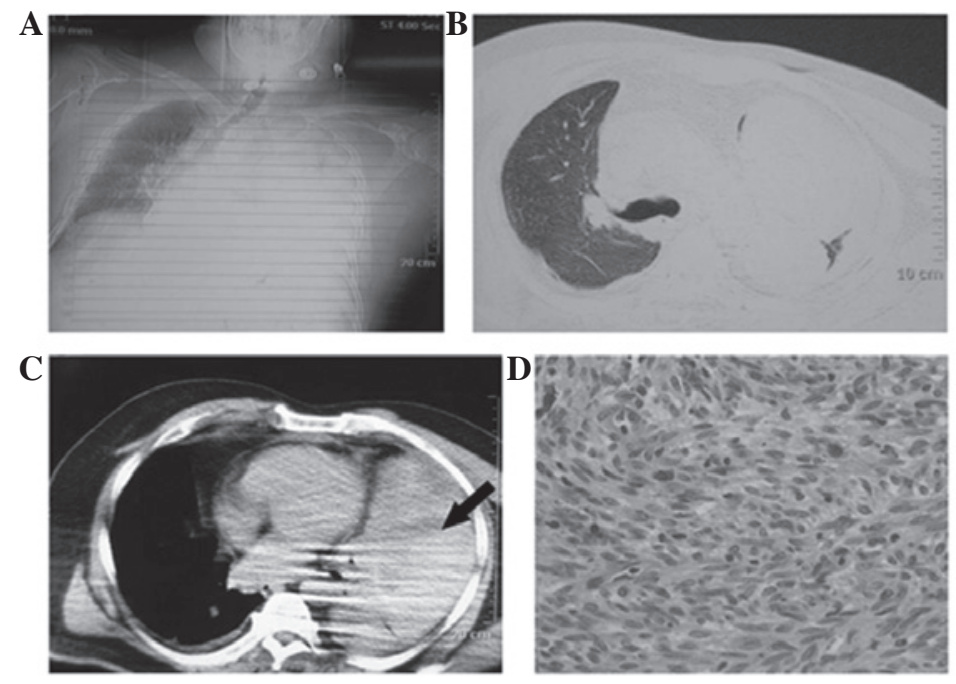

Figure 1. Pre-operative computed tomography showing (A) left lung atelectasis, (B) a patchy shadow in the lower lobe of the right lung and (C) an irregular soft-tissue shadow in the left side of the chest. (D) Specimen showing synovial sarcoma composed of spindle cells (H\&E staining; magnification, x400).

tissue, with hemorrhage, necrosis, collagen degeneration and a diffuse spindle cell proliferative zone. Following morphological and immunohistochemical analysis, the patient was diagnosed with synovial sarcoma. The tumor cells exhibited immunohistochemical positivity for vimentin, epithelial membrane antigen and calponin, but negativity for S-100 and cluster of differentiation 34 (Fig. 1D). The patient's pain was relieved after the surgery and the follow-up chest CT showed that the mass was significantly reduced in size (Fig. 2A). The patient went home after the surgery on May 17, 2011, without further treatment.

After 2 months, a follow-up chest CT scan showed local recurrence (Fig. 2B). Between July 15, 2011, and September 23, 2011, the patient received four cycles of chemotherapy consisting of $7.5 \mathrm{mg} / \mathrm{m}^{2}$ Endostar intravenous guttae (ivgtt) on days 1-14 and $1.0 \mathrm{~g} / \mathrm{m}^{2}$ ifosfamide ivgtt on days $1-6$. During the treatment, the imaging evaluation was of a partial response (Fig. 2C). The main adverse effects of the treatment were grade IV nausea, vomiting and dizziness.

Between November 14, 2011, and February 2, 2012, due to chest pain and disease progression on chest CT (Fig. 2D), the patient was administered 3 cycles of chemotherapy $\left(250 \mathrm{mg} / \mathrm{m}^{2}\right.$ pemetrexed ivgtt on days $1-8$ and $7.5 \mathrm{mg} / \mathrm{m}^{2}$ Endostar ivgtt on days 1-14). The subsequent therapeutic evaluation was of stable disease (Fig. 2E). On February 2, 2012, the patient began to receive sunitinib $\left(375 \mathrm{mg} / \mathrm{m}^{2}\right)$ as maintenance therapy. On July 23, 2013, the patient succumbed to respiratory and circulatory failure due to tumor progression.

\section{Discussion}

In the present study, sunitinib was administered to the patient following combined treatment with chemotherapy and Endostar. To the best of our knowledge, this is the first reported use of sunitinib for maintenance therapy in pleural synovial sarcoma and it achieved a good curative effect. An imaging examination showed that the tumor size was significantly reduced and the patient's survival time was 25 months when the tumor recurred after surgery. The main adverse effects during treatment were hypertension and neutropenia.
Although the treatment was effective, the patient's quality of life was poor. From March 2012 onwards, the patient was unable to function independently due to chest pain. The sarcoma had a brittle texture, and as a result any careless action may have lead to tumor rupture and severe pain. The experience of bleeding greatly affected the psychology of the patient, who became afraid and depressed due to the pain and had to use a Fentanyl transdermal system for a prolonged period of time, with a maximum dose of $134.4 \mathrm{mg}$. In April 2013, the patient accepted a nerve block and nerve ablation, but the procedures did not produce the desired therapeutic effect.

The incidence of pleural synovial sarcoma has been shown to be increasing and the most common therapy is a surgical resection $(6,7)$, followed by chemotherapy. In general, synovial sarcomas are chemosensitive to ifosfamide and doxorubicin, but there is no established gold standard for the treatment of pleural synovial sarcoma. The rarity of synovial sarcoma means that there is no consensus on the role of chemotherapy in preventing metastases and improving survival.

However, antiangiogenic therapy in general shows promise for the treatment of malignancy, tumor invasion and metastasis of a number of different types of cancer. O'Reilly et al first identified endostatin in hemangioendothelioma cells as an antiangiogenic molecule (8). The targets of endostatin are the newly formed capillary endothelial cells, which are intimately associated with tumors (9). Endostar (YH-16) is a novel recombinant human endostatin, which has a broad spectrum of antiangiogenic activity; its mechanism of action is to stimulate the migration of endothelial cells by inhibiting the formation of blood vessels and tumor neovascularization, thus blocking the nutrient supply of the tumor, inhibiting tumor proliferation and metastasis. Endostar also exhibits anti-xenograft activity against human tumor cells (SPC2A4 lung cancer, SGC7901 gastric cancer, HeLa cervical cancer, and SMMC27721 and Bel7402 hepatoma cells). Endostar has also shown antitumor activity in vitro in a murine tumor model of broad-spectrum cancers (such as S180 sarcoma and H22 hepatocellular carcinoma cells). 
A

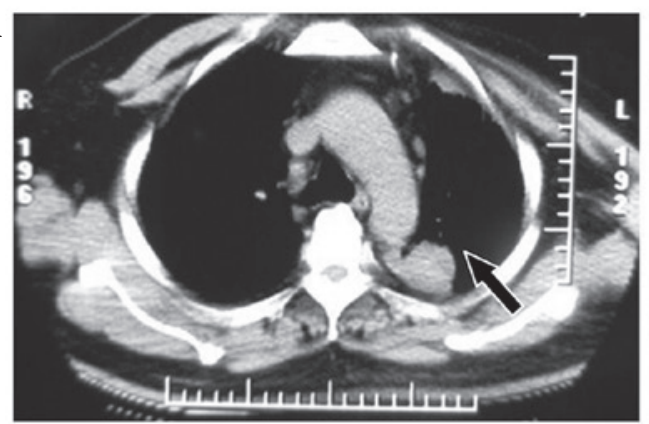

$\mathbf{B}$

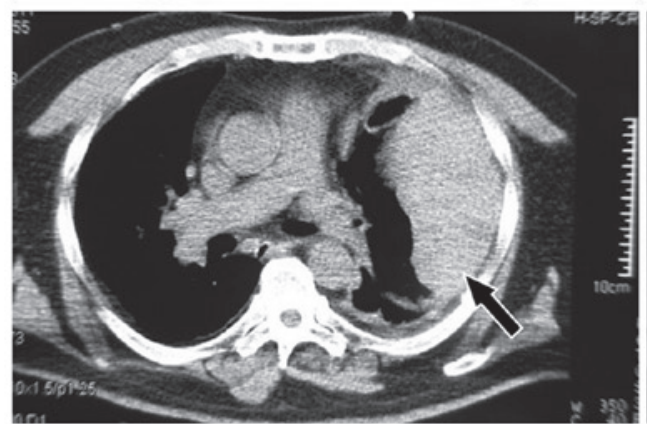

C

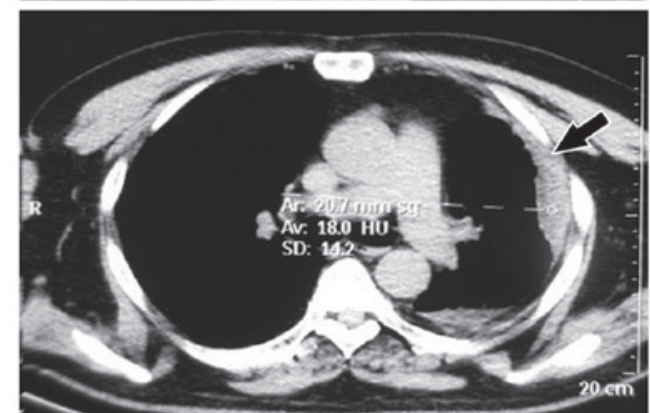

D

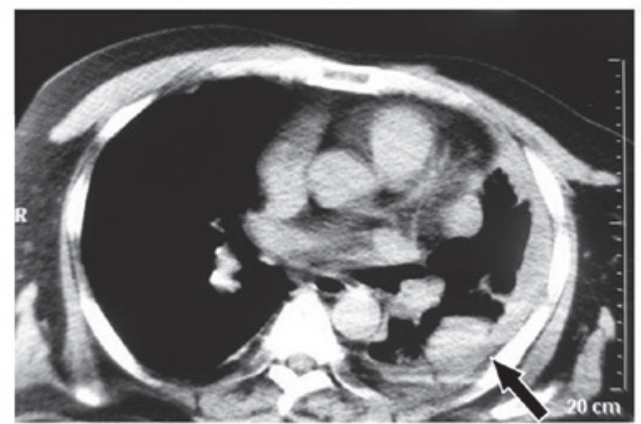

$\mathbf{E}$

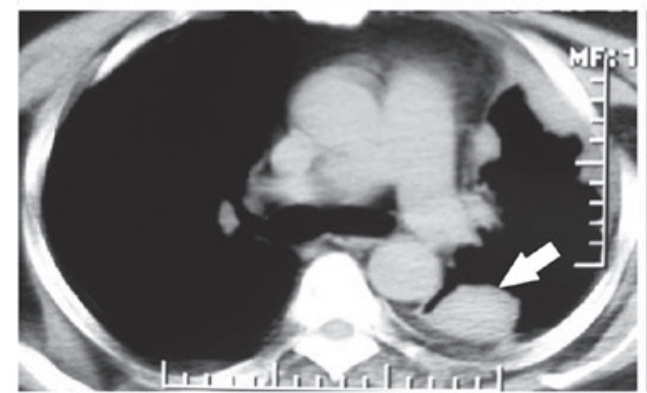

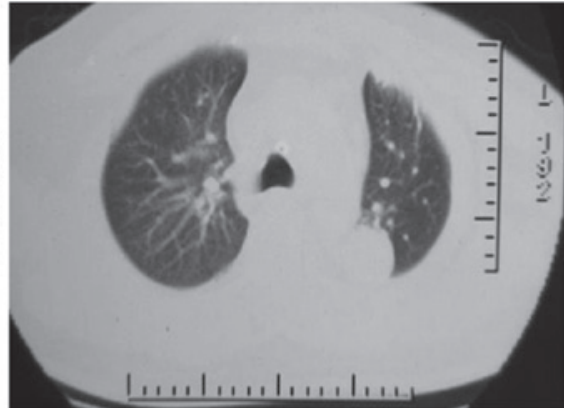
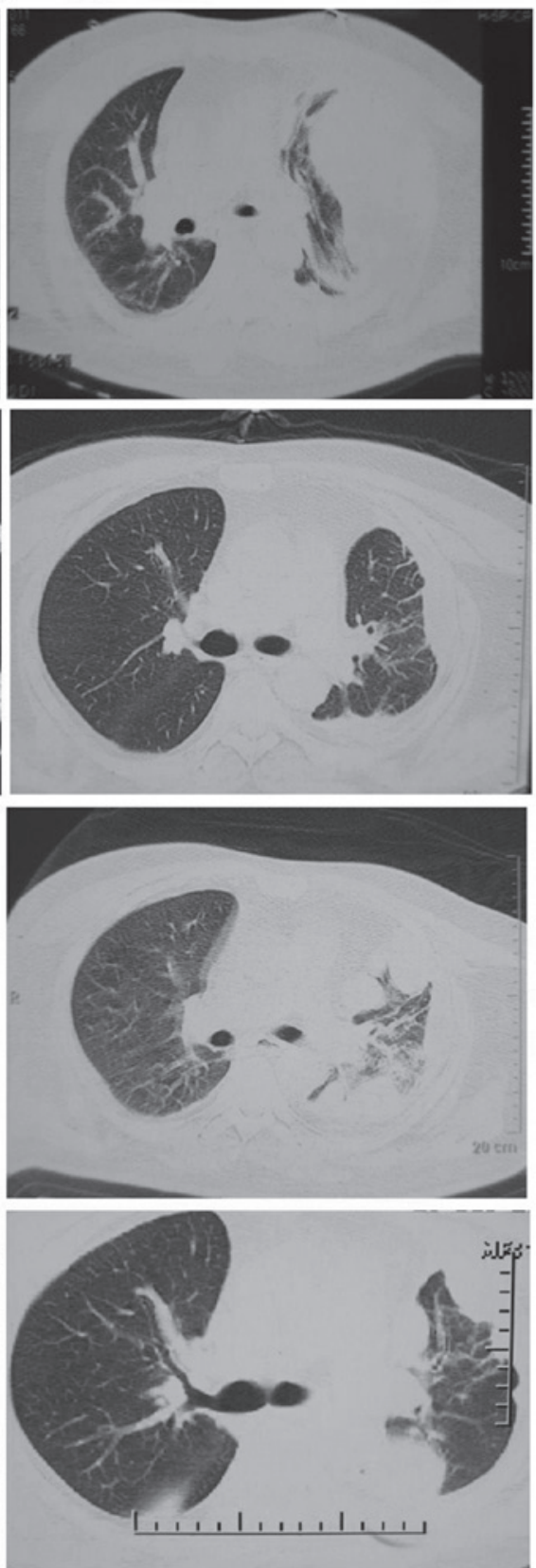

Figure 2. Chest (computed tomography) CT at the different stages of treatment. (A) Post-operative CT showing a significant reduction in the tumor mass. (B) Left pleural effusion, bilateral pleural thickening calcification, left huge pleural tumor formation. (C) After 4 cycles of Endostar + ifosfamide, the imaging evaluation was a partial response; left pleural fluid. (D) High-density shadows in the left mediastinum and pleural inner circular lesions characteristic of bilateral pulmonary infection (December 23, 2011). (E) The therapeutic evaluation after several cycles of Endostar + pemetrexed was of stable disease. Arrows indicate pleural tumor.

Sunitinib is an orally active tyrosine kinase inhibitor (TKI) that affects various signaling cascades involved in antiangiogenesis and tumor proliferation, including vascular endothelial growth factors and platelet-derived growth factors. The drug is used in the first-line therapy of clear cell renal carcinoma and imatinib-resistant gastrointestinal stromal tumors, and 
is also used to treat numerous other types of solid tumors, including breast, lung, prostatic and colorectal cancer (10-13). For example, the efficacy of the TKIs imatinib (Gleevec) and sunitinib (Sutent) in patients with advanced gastrointestinal stromal tumors (GISTs) has been demonstrated by observations in the clinic, a disease for which there were previously no highly effective treatment options for metastatic disease. There is a paucity of data in the literature regarding the role of sunitinib in synovial sarcoma, therefore the present study investigated its efficacy in synovial sarcoma. One previous report was located in the literature about a patient with synovial sarcoma, who also had a non-GIST and stable disease following 16 weeks of therapy with sunitinib (14).

In conclusion, it is worth considering using Endostar and targeted therapy following chemotherapy to optimize the treatment of pleural synovial sarcoma. Sunitinib can be used in pleural synovial sarcomas as maintenance therapy. The optimal dose and treatment time with Endostar and sunitinib remains to be established in clinical trials with more patients. Sunitinib is a useful practical adjuvant for the management of pleural synovial sarcomas.

\section{Acknowledgements}

This study is supported by the fund of the Natural Science Foundation of Heilongjiang (grant no. D201223).

\section{References}

1. Mankin HJ and Hornicek FJ: Diagnosis, classification, and management of soft tissue sarcomas. Cancer Control 12: 5-21, 2005.

2. Aubry MC, Bridge JA, Wickert R and Tazelaar HD: Primary monophasic synovial sarcoma of the pleura: Five cases confirmed by the presence of SYT-SSX fusion transcript. Am J Surg Pathol 25: 776-781, 2001.
3. Gaertner E, Zeren EH, Fleming MV, Colby TV and Travis WD: Biphasic synovial sarcomas arising in the pleural cavity. A clinicopathologic study of five cases. Am J Surg Pathol 20: 36-45, 1996.

4. Jawahar DA, Vuletin JC, Gorecki P, Persechino F, Macera M and Magazeh P: Primary biphasic synovial sarcoma of the pleura. Respir Med 91: 568-570, 1997.

5. Nicholson AG, Goldstraw P and Fisher C: Synovial sarcoma of the pleura and its differentiation from other primary pleural tumours: A clinicopathological and immunohistochemical review of three cases. Histopathology 33: 508-513, 1998.

6. Loscertales J, Triviño A, Gallardo G and Congregado M: Primary monophasic synovial sarcoma of the pleura: Diagnosis and treatment. Interact Cardiovasc Thorac Surg 12: 885-887, 2011.

7. Nishiyama N, Iwata T, Izumi N, Tsukioka T, Nagano K and Suehiro S: Aggressive repeat surgery for a recurrent synovial sarcoma in the pleura. Ann Thorac Cardiovasc Surg 15: 46-49, 2009.

8. O'Reilly MS, Boehm T, Shing Y, et al: Endostatin: an endogenous inhibitor of angiogenesis and tumor growth. Cell 88: 277-285, 1997.

9. Zhuo W, Chen Y, Song X and Luo Y: Endostatin specifically targets both tumor blood vessels and lymphatic vessels. Front Med 5: 336-340, 2011

10. Cardoso F, Canon JL, Amadori D, et al: An exploratory study of sunitinib in combination with docetaxel and trastuzumab as first-line therapy for HER2-positive metastatic breast cancer. Breast 21: 716-723, 2012.

11. Corn PG, Song DY, Heath E, et al: Sunitinib plus androgen deprivation and radiation therapy for patients with localized high-risk prostate cancer: results from a multi-institutional phase 1 study. Int J Radiat Oncol Biol Phys 86: 540-545, 2013.

12. Modest DP, Camaj P, Heinemann V, et al: KRAS allel-specific activity of sunitinib in an isogenic disease model of colorectal cancer. J Cancer Res Clin Oncol 139: 953-961, 2013.

13. Waqar SN, Gopalan PK, Williams K, Devarakonda S and Govindan R: A phase I trial of sunitinib and rapamycin in patients with advanced non-small cell lung cancer. Chemotherapy 59: 8-13, 2013.

14. George S, Merriam P, Maki RG, et al: Multicenter phase II trial of sunitinib in the treatment of nongastrointestinal stromal tumor sarcomas. J Clin Oncol 27: 3154-3160, 2009. 